\title{
A COMPARATIVE STUDY OF STEREOCHEMICAL EFFECTS OF ANTI-PROSTATE AGENTS BY MOLECULAR DOCKING
}

\section{OLUWASEUN S OSANYINPEJU ${ }^{1}$, ROQIA BASHARY ${ }^{1,2}$, AMIT MITTAL ${ }^{1}$, MANISH VYAS $^{1}$, SURENDRA KUMAR NAYAK $^{1}$, GOPAL L. KHATIK ${ }^{1 *}$}

${ }^{1}$ Department ofPharmaceutical Chemistry, School of Pharmaceutical Sciences, Lovely Professional University, Jalandhar-Delhi G.T. Road, Phagwara - 144 411, Punjab, India. ${ }^{2}$ Department of Pharmaceutical Chemistry, Kabul University, Afghanistan. Email: gopal.16803@lpu.co.in

Received: 04 March 2018, Revised and Accepted: 11 April 2018

ABSTRACT

Objective: A comparative study of anti-prostate agents to investigate the stereochemical influences on binding affinity by molecular docking.

Methods: Structures of enantiomers (R and S stereoisomers) for known anti-prostate cancer (PCa) agents were drawn using ChemBioDraw 2D software. Thereafter, they were converted to 3D structures using the ChemBioDraw 3D software in which they were subjected to energy minimization using the MM2 method and then saved as PDB extension files which can be accessed using the ADT interface. AutoDock Vina (ADT) 1.5.6 software version was used for molecular docking study.

Results: A total of 12 different anti-PCa agents were selected and drawn including well-known drug $R$-bicalutamide. All molecules showed the binding affinity with respect to the nature of stereochemistry. $R$-stereoisomers showed better interaction as well as binding affinity toward $1 \mathrm{z} 95$ (mutated androgen receptor protein involved in the progression of PCa) whereas their S-stereoisomers were found inferior in comparison.

Conclusion: This study showed that CB1-R and $R$-bicalutamide (with $R$-stereochemistry) were better in binding affinity comparative to their counterpart CB1-S and $S$-Bicalutamide (with $S$-stereochemistry). All the selected anti-PCa agents were showing the effect of stereochemical center; therefore, we must choose the right kind of stereochemistry while planning to develop the newer anti-PCa agents.

Keywords: Stereoisomer, Molecular docking, Anti-prostate agent, AutoDock Vina, Androgen.

(C) 2018 The Authors. Published by Innovare Academic Sciences Pvt Ltd. This is an open access article under the CC BY license (http://creativecommons. org/licenses/by/4. 0/) DOI: http://dx.doi.org/10.22159/ajpcr.2018.v11s2.28587

\section{INTRODUCTION}

Prostate cancer (PCa) has been described as a disease that affects the prostate region of the male genital organ due to the proliferation of androgen receptors and hormones present in the prostate organ [1]. Research shows in the preventive medicine reports that PCa continues to disproportionately affect African-American men in terms of mobility and mortality [2]. Furthermore, various examination studies, knowledge, beliefs, and behaviors on PCa screening [3] and recent researches also evaluate the effect of interventions that focus on enhancing the screening and about decision-making among AfricanAmerican men $[4,5]$.

As described and illustrated earlier, PCa is a leading cause of death in men. Globally, it is the most diagnosed/screened among men AutoDock Vina between the ages of 65 and 74 years [2]. The prediction or diagnosis differs greatly, but highly dependent on a number of factors such as stage of diagnosis, race, and age. Currently, the treatment of $\mathrm{PCa}$ includes androgen deprivation, surgery, radiation, endocrine therapy, radical prostatectomy, and chemotherapy [6]. Drugs like flutamide (Eulexin), hydroxyflutamide (Active form of flutamide), bicalutamide (Casodex), nilutamide, and enzalutamide (formerly called MDV3100) (Xtomdi) are all non-steroidal androgen receptor antagonists which were approved for the treatment of PCa [7].

Recent years, stereochemistry has gained great attention in pharmaceutical industries and regulatory authorities [8]. Stereochemistry is mostly dealt with compounds which possess chiral centers. Chiral compounds which are not superimposable in their mirror images are known as enantiomers and have opposite rotation of the direction of plane polarized light. $R$ and $S$ stereoisomers are the enantiomers represent the absolute stereochemistry of stereocenter in 3D structure. These enantiomers behave differently in interaction with chiral environments such as enzymes, receptors, and transporters. Generally, they differ in chemical and pharmacological properties such as bioavailability, the rate of metabolism, metabolites, excretion, potency, and selectivity for receptors, enzymes, and transporters [9]. $50 \%$ of marketed drugs are chiral and out of that $50 \%$ are available as racemate (an equal mixture of both enantiomers) mixtures [10]. The most important thing here is that these two enantiomers may have same pharmacological action, for example, ofloxacin (R-enantiomer) and levofloxacin (S-enantiomer) which are both antimicrobials of quinolones [11]. One enantiomer may be active and the other may be inactive, the examples are (S)-albuterol, a $\beta 2$-adrenergic receptor agonist for treatment of asthma, and (S)-omeprazole, a proton pump inhibitor for treatment of gastroesophageal reflux has been shown to be superior to their racemic formulations in clinical trials cause their $R$-enantiomers are inactive [9]. One of them may be having some undesirable effects, like $S$-timolol, a $\beta$-blocker agent can cause severe bronchoconstriction, while R-isomer did not cause, due to this it is marketed as pure $R$-enantiomer [12]. Hence, to achieve better results in therapy and prevent harmful effects of unwanted enantiomer, the availability and isolation of a single enantiomer have become an important issue in pharmaceuticals, nowadays.

\section{METHODS}

Computer-aided drug design, especially molecular docking, is a very useful method to find out the potent molecule without investing extreme effort along with funds in research [13-17]. ADT 1.5.6 software [18] is 
used by us to examine the activity in terms of binding affinity (Kcal/ $\mathrm{mol}$ ), and thereafter, the outcomes were compared in binding affinity score for best-docked conformation. The structures of various known anti-PCa agents were drawn with their stereochemistry with the help of ChemBiodraw ultra and further converted to the 3D structure using ChemBiodraw 3D. All the designed structures were optimized by energy minimization using MM2 method [19] and converted to readable format at the ADT interface. For a comparative study of stereoisomers of the anti-PCa agents, a protein 1z95 was selected and downloaded from protein data bank [20]. The outcomes of results were analyzed by AutoDock Vina (ADT) result which reveals close contact, hydrogen bond, hydrophilic, and hydrophobic interactions.

\section{RESULTS AND DISCUSSION}

The main purpose of this study is to find out the stereochemical effect of anti-PCa agents which are used the treatment of PCa or recently investigated. The molecules were selected from the available literature having a different class of compounds, that is, bicalutamide [21], propionamides [22], and thioxoimidazolidinones [23] as shown in Fig. 1.

All structures were drawn through ChemBioDraw 2D software (both $\mathrm{R}$ and $\mathrm{S}$ or RR and SS) to study the stereochemical effect and then converted into 3D structures using ChemBioDraw 3D software an later saved as pdb files so that it can be accessed by the ADT software. Androgen protein receptor was downloaded from the protein data bank site rcsb.org specifically protein with code $1 \mathrm{z} 95$ for this study.

All the selected molecules were analyzed using the ADT molecular modeling software. Initially, the protein validation has been done by extraction of ligand (Fig. 2). Preparations of ligands were done by the addition of polar hydrogens, detecting root, and converting it to pdbqt extension file, while $1 \mathrm{z} 95$ protein was prepared by removing water molecules, repairing missing atoms, adding polar hydrogens only, and subsequently adding the Kollman charges. Further, the grid box was generated keeping the ligand as a center (Fig. 3). From grid output file, the configuration file "conf.txt" was prepared and command prompt was used for ADT molecular docking by giving the command "program files\the scripps research institute\vina\vina. exe --config conf.txt --log log.txt." It generated the output file with the docking score or binding affinity (Kcal/mol). Similarly, all twelve molecules were studied and their binding affinities are shown in Table 1.

The study was based on twelve different molecules containing one or more stereocenter. Binding affinities represented in Table 1 revealed that stereochemistry played a critical role and considered for better interaction with the $1 \mathrm{z} 95$ protein. This was observed while comparison of the binding affinities of all $R$-stereoisomers with their $S$-stereoisomers. To know the effect of stereochemistry and various substituents, all the interactions of protein 1 z95 were analyzed in detail. Different amino acid residues are found to be involved in the interactions at binding sites include ILE899, ASN705, LEU704, THR877, ARG752, MET745, MET895, GLN711, and LEU741. The aforementioned amino acids can be classified on the basis of their polarity, hydrophobicity, and charge such as ILE899, LEU704, MET745, MET895, and LEU741 are hydrophobic in nature, while ASN705, THR877, and GLN711 are polar amino acids present in the protein receptor $1 \mathrm{z} 95$, therefore, represent similar kind of interaction with the molecule of interest.

Both $R$ or $R R$ and $S$ or $S S$ were studied for their interactions with the $1 \mathrm{z} 95$ protein. These results suggested the essence of stereocenter on the binding affinity of the existing anti-PCa agents. In Table 1 , all propanamide and thioxoimidazolidinone derivatives having $\mathrm{R}$ and $\mathrm{R}, \mathrm{R}$ stereocenter showed more binding affinity than the $S$ and $S, S$ stereocenter ligands. Among them, molecules with higher binding affinity were discussed herein in detail. Molecule CA1-R ( $R$-bicalutamide) and CA1-S ( $S$-bicalutamide) were found to be with

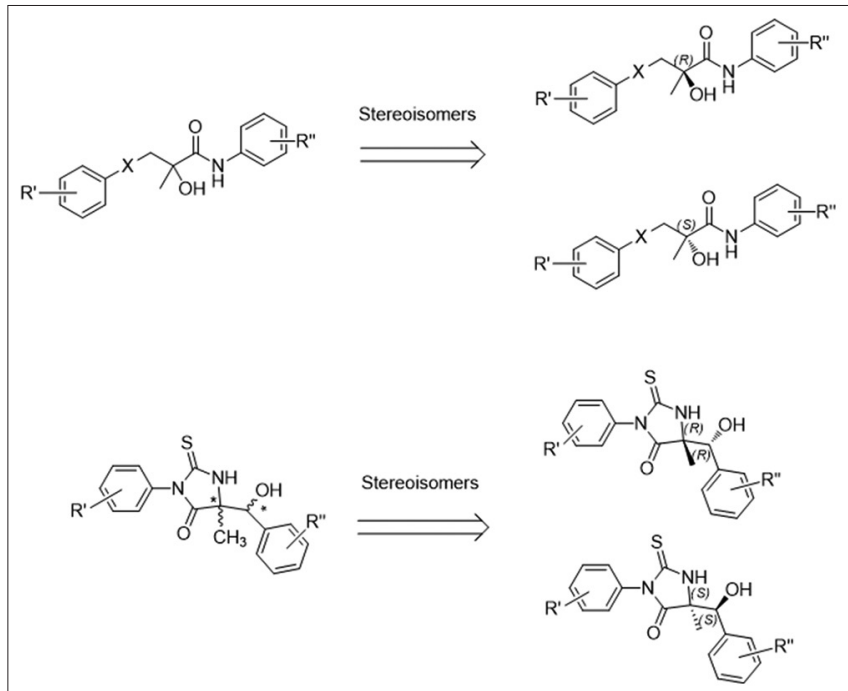

Fig. 1: Selected anti-prostate cancer agents for the comparative study of stereochemical effect

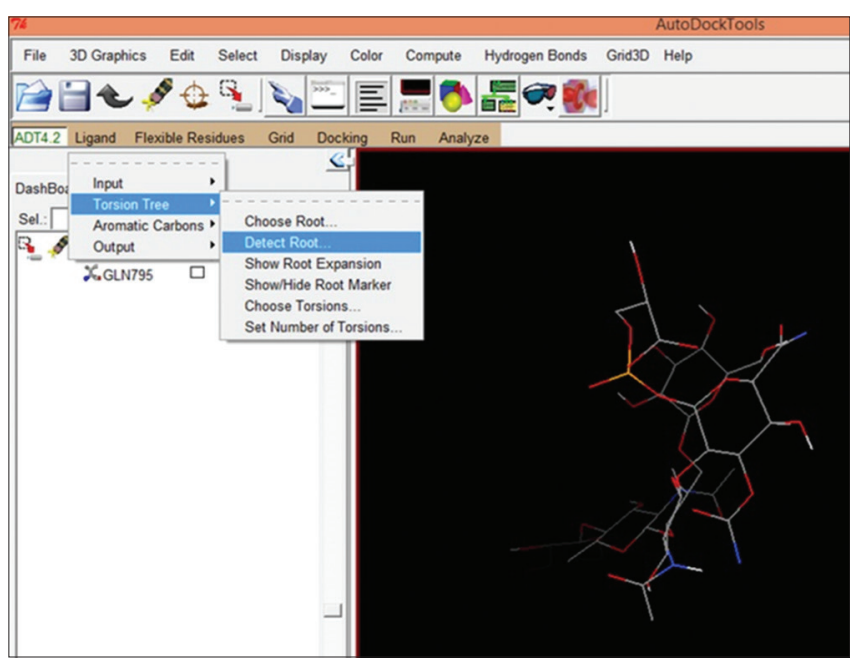

Fig. 2: Extracted and preparation of ligand from $1 \mathrm{z} 95$ protein

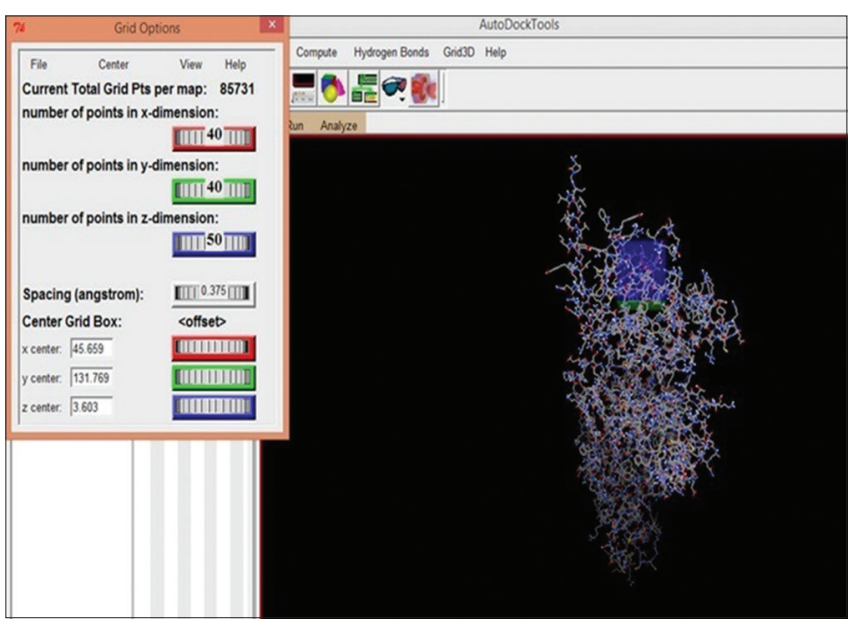

Fig. 3: Set grid box on 1z95 protein as a macromolecule

$-11.3 \mathrm{Kcal} / \mathrm{mol}$ and $-10.3 \mathrm{Kcal} / \mathrm{mol}$ binding affinity, respectively, to $1 \mathrm{z} 95$ protein. 


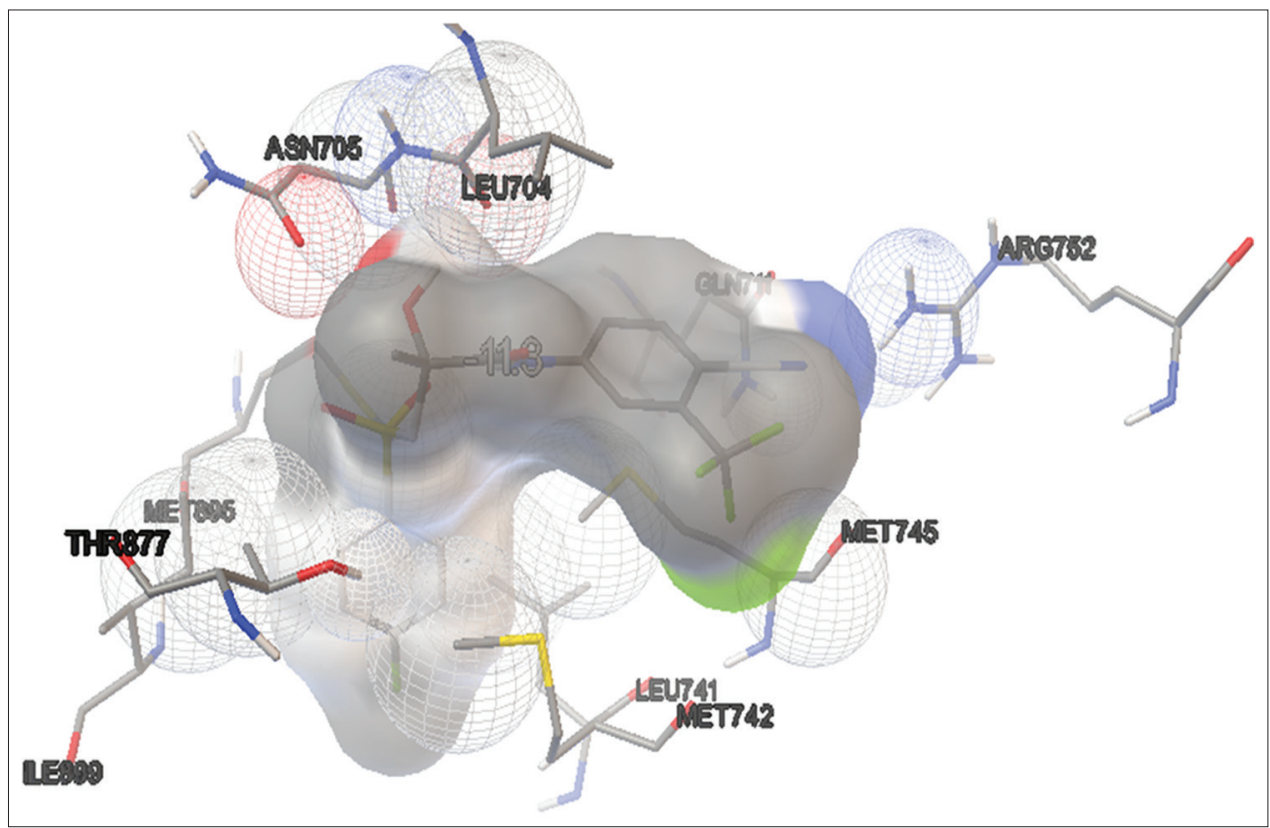

Fig 4: Interactions between ligand CA1-R (R-stereoisomer) and protein $1 \mathrm{z95}$

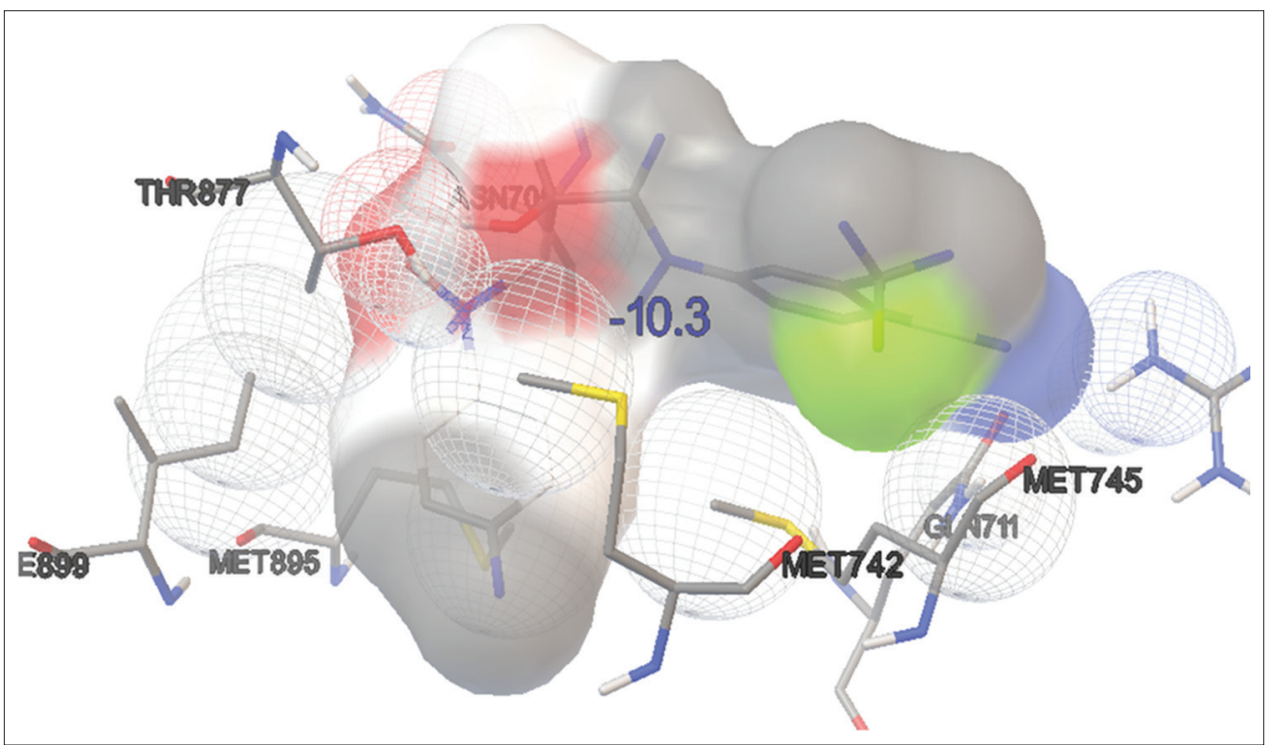

Fig. 5: Interactions between ligand CA1-S (S-stereoisomer) and protein $1 \mathrm{z95}$

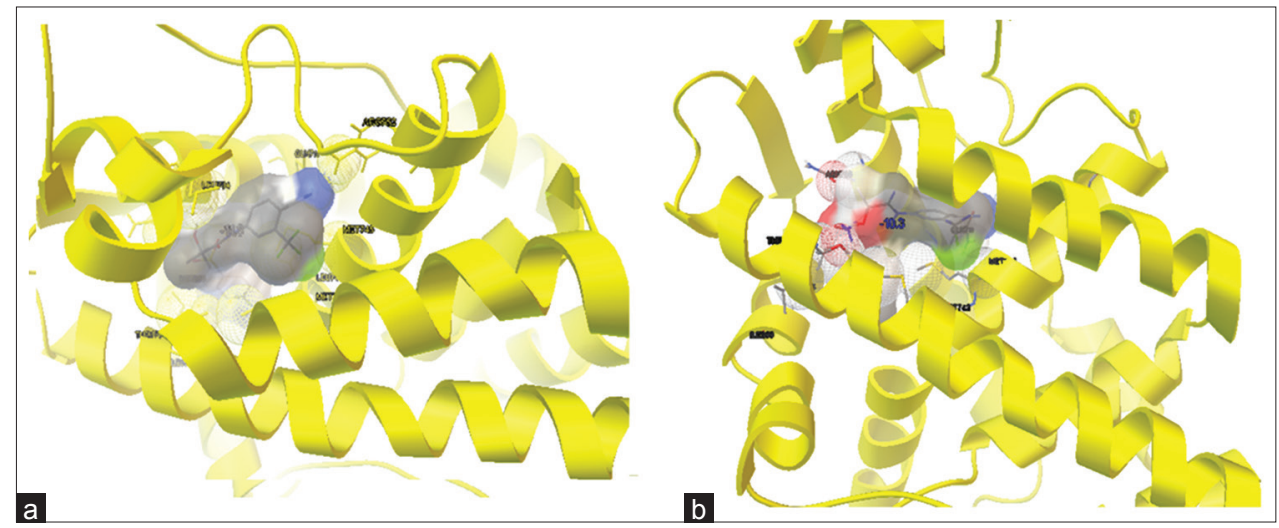

Fig. 6: (a) Ligand CA1-R (R-stereoisomer) and (b) CA1-S (S-stereoisomer) with ribbon structure of protein $1 \mathrm{z95}$ 
Table 1: Selected ligands with affinity

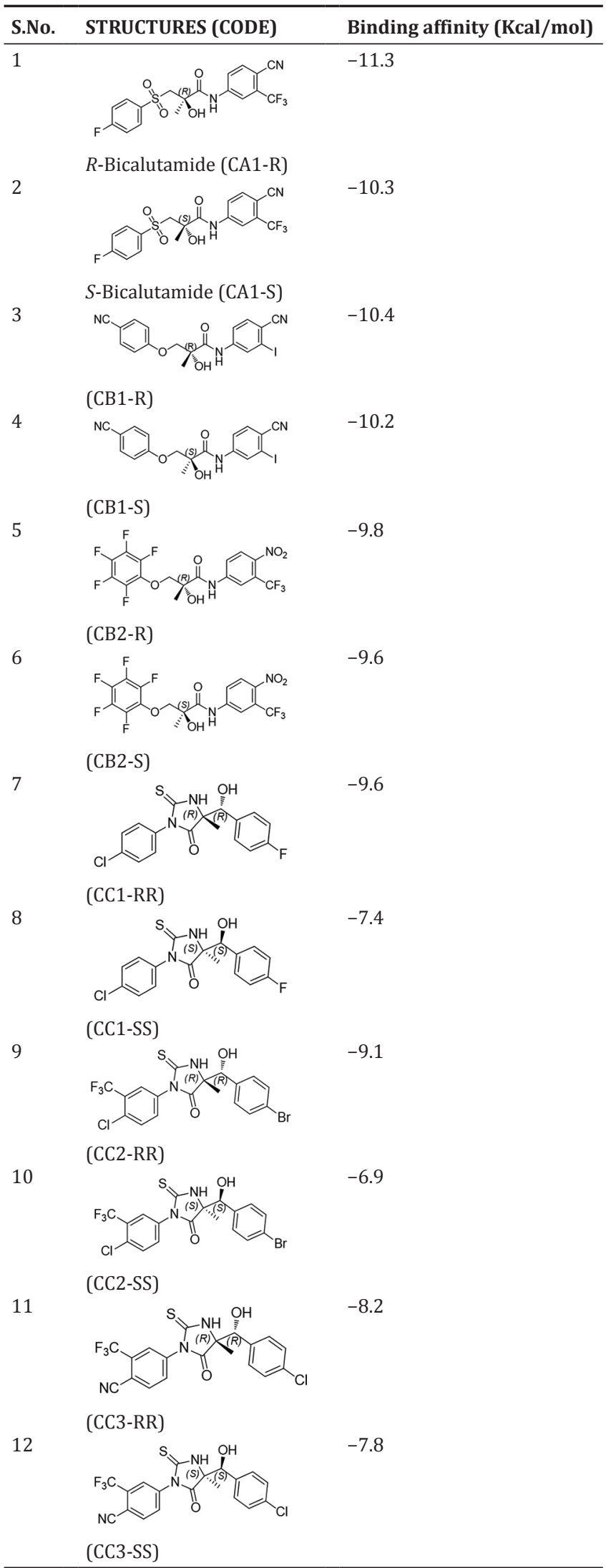

A detail observations showed that CA1-R (R-bicalutamide) has favorable interaction with 1z95 androgen receptor (Fig. 4). The amine group of ARG752 of protein $1 z 95$ has hydrogen bonding with the cyano group

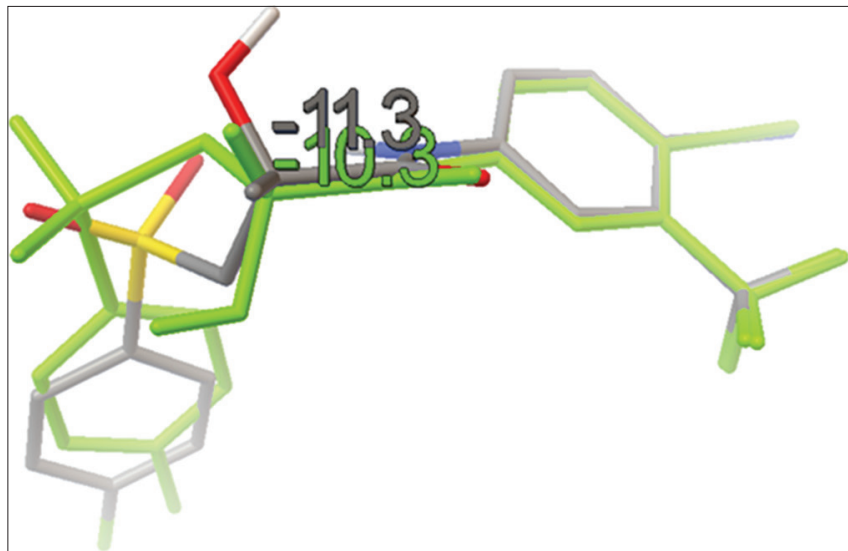

Fig. 7: Overlay of ligands CA1-R (R-stereoisomer) and CA1-S (S-stereoisomer) with their binding affinity

of CA1-R ( $R$-bicalutamide), similar interaction showed by cyano group with MTH745 and GLU711. The carbonyl group of the ASN705 residue of the $1 \mathrm{z} 95$ is involved in a hydrogen bonding with the chiral hydroxyl group and sulphone group of CA1-R ( $R$-bicalutamide). The interactions between the protein (1z95) and ligand CA1-S ( $S$-bicalutamide) are less favorable (Figs. 5 and 6). Herein, also amine group of the ARG752 residue of the receptor protein is involved in hydrogen bonding with the cyano group of CA1-S ( $S$-bicalutamide), while chiral hydroxyl group does not involve in hydrogen bonding resulting in low binding affinity. The overlay of CA1-S (S-bicalutamide) and CA1-R (R-bicalutamide) depicted in Fig. 7 showed the importance of chiral hydroxyl group which is opposite in case of CA1-S (S-bicalutamide), therefore, lacking hydrogen bonding and resulting low binding affinity.

\section{CONCLUSION}

The evolution of PCa occurs as a result of the overexpression of androgen receptor. There are several therapies available to treat $\mathrm{PCa}$, but the most commonly used is chemotherapy include non-steroidal drugs. These drug molecules have a good involvement of hydroxyl group with particular stereochemistry. Thus, the present study is undertaken to investigate the role of the stereochemistry of bicalutamide, propanamide, and thioxoimidazolidinones as anti-PCa agents.

ADT molecular software was used for molecular docking of all stereoisomers on $1 \mathrm{z} 95$ (Androgen receptor) protein. Binding affinities revealed that molecule with score shows that R-stereochemistry is very important at the binding site resulting in higher binding affinity than its S-stereoisomer. Therefore, it could be suggested that while designing the novel anti-PCa agents, we must consider the stereochemistry at the stereocenter.

\section{ACKNOWLEDGMENTS}

The author is grateful to SERB - Department of Science and Technology Government of India for research funding.

\section{CONFLICTS OF INTEREST}

The author's declared that they have no conflicts of interest.

\section{REFERENCES}

1. Guyton AC, Hall JE. Textbook of Medical Physiology. $11^{\text {th }}$ ed. Philadelphia, PA: W.B. Saunders; 2006. p. 996-9.

2. Ferlay J, Shin HR, Bray F, Forman D, Mathers C, Parkin DM. Estimates of worldwide burden of cancer in 2008: GLOBOCAN 2008. Int J Cancer 2010;127:2893-917.

3. Dumitrescu R, Cotarla I. Understanding breast cancer risk-where do we stand in 2005? J Cell Mol Med 2005;9:208-21.

4. Steele CB, Miller DS, Maylahn C, Uhler RJ, Baker CT. Knowledge, attitudes, and screening practices among older men regarding prostate 
cancer. Am J Public Health 2000;10:1595-600.

5. Volk RJ, Hawley ST, Kneuper S, Holden EW, Stroud LA, Cooper CP, et al. Trials of decision aids for prostate cancer screening: A systematic review. Am J Prev Med 2007:5:428-34.

6. Kolvenbag GJ, Iversen P, Newling DW. Antiandrogen monotherapy: A new form of treatment for patients with prostate cancer. Urology 2001;58:16-22.

7. Kaur P, Khatik GL. Advancements in non-steroidal antiandrogens as potential therapeutic agents for the treatment of prostate cancer. Mini Rev Med Chem 2016;16:531-46.

8. Hutt AJ, Grady JO. Drug chirality: A consideration of the significance of the stereochemistry of antimicrobial agents. J Antimicrob Chemother 1996;37:7-32.

9. McConathy J, Owens MJ. Stereochemistry in drug action. Prim Care Companion J Clin Psychiatr 2003;5:70-3.

10. Caldwell J. The importance of stereochemistry in drug action and disposition. J Clin Pharmacol 1992;32:1925-9.

11. Edlund C, Sjostedt S, Nord CE. Comparative effects of levofloxacin and ofloxacin on the normal oral and intestinal microfiora. Scand J Infect Dis 1997;29:383-6.

12. Drayer DE. Pharmacodynamics and pharmacokinetic differences between drug enantiomers in humans: An overview. Clin Pharmacol Ther 1986;40:125-33.

13. Chaurasiya S, Kaur P, Nayak SK, Khatik GL. Virtual screening for identification of novel potent EGFR inhibitors through AutoDock Vina molecular modeling software. J Chem Pharm Res 2016;8:353-60.

14. Muthukala B, Kanakarajan S, Kamalanathan A. In silico docking of quercetin compound against the HeLa cell line proteins. Int J Curr
Pharm Res 2015;7:13-6.

15. Kaur P, Khatik GL. Identification of novel 5-styryl-1,2,4-oxadiazole/ Triazole derivatives as the potential antiandrogens. Through molecular docking study. Int J Pharm Pharm Sci 2016;8:72-7.

16. Kaur K, Kaur P, Mittal A, Nayak SK, Khatik GL. Design and molecular docking studies of novel antimicrobial peptides using AutoDock molecular docking software. Asian J Pharm Clin Res 2017;10:28-31.

17. Bhardwaj S, Khatik GL, Kaur P, Nayak SK. Computer aided drug design through molecular docking: Identification of selective COX-2 inhibitors as potential NSAIDs. J Pharm Res 2017;11:604-8.

18. Trott O, Olson AJ. AutoDock Vina: Improving the speed and accuracy of docking with a new scoring function, efficient optimization, and multithreading. J Comput Chem 2010;31:455-61.

19. Energy Minimizations were Performed MM2 Interface Program on ChemBio3D Ultra 12.0, and Structures were Drawn by ChemBioDraw Ultra 12.0. Cambridge: Cambridge Soft.; 1985.

20. $1 Z 95$ Protein. Available from: http://www.rcsb.org/pdb/explore. do?pdbId=1z95. [Last accessed on 2017 Oct 22].

21. Cockshott ID. Bicalutamide: Clinical pharmacokinetics and metabolism. Clin Pharmacokinet 2004;43:855-78.

22. Bohl CE, Wu Z, Chen J, Mohler ML, Yang J, Hwang DJ, et al. Effect of B-ring substitution pattern on binding mode of propionamide selective androgen receptor modulators. Bioorg Med Chem Lett 2008;18:5567-70.

23. Khatik GL, Kaur J, Kumar V, Tikoo K, Venugopalan P, Nair VA. Aldol derivatives of Thioxoimidazolidinones as potential anti-prostate cancer agents. Eur J Med Chem 2011;46:3291-301. 